\title{
Summarization and Negative Reviews Opinion Mining of Multiple User Reviews in Text Domain
}

\author{
Anita K. Bodke \\ Student, LGNSCOE \\ Mahiravani, Nashik
}

\author{
M. G. Bhandare \\ Professor, LGNSCOE \\ Mahiravani, Nashik
}

\begin{abstract}
As all uses online services so it become tedious job to kind opinion about needed things likes,publication,restaurant etc.so here develop system which take input reviews and tips(microreview) from different sites and provide user a compact and informative set of review. Problem of selection reviews which cover maximum number of tips is NP-hard, so provide a maximum solution, use greedy approach to solve problem. Also provide user a reason behind negative review. For this develop our own algorithm. For the project data collect from webKB,Fouresquare.com,yelp.com.Proposed system select here tips for selecting informative review because tips are highly concise, authentic(user place it when he/her check in at that place),content relevant data.
\end{abstract}

\section{General Terms \\ LDA, N-gram}

\section{Keywords}

Sentimental, syntactic, semantic similarity, Review, Micro review, coverage, efficiency.

\section{INTRODUCTION}

Now days Data handling and management is important. Micro-review new type of online review content.Review is basically an assessment of a publication, service, company or performance. Want to find alternative source of content for the readers to search desired review .Information provided must be compact and comprehensive.WebKB Yelp.com is a widespread website for cafe reviews, these websites provide introductory information to user. There are several needs and problems to deal with online reviews, because reviews are lengthier and less attentive on topic, whose content may not be just relevant to the product or service being reviewed. As People know in this digital Technology world new type of sites are available which provide new concept called as Tips or Micro-review .Blogging services that allow users to register it, representing their current Setting, Development, situation activity. Micro-review sites provide authentic tips and if tip unauthentic it get filter out by sites. This problem (select top reviews that match to micro review) is of interest to any online site or mobile application that wishes to showcase a small number of reviews. All know todays more number of customer uses mobile but they got less time for read more than 100 reviews lengthy and verbose, so inclusion of micro review is better option for this type of customer.

\section{LITERATURE SURVEY}

[7]Selecting a characteristic set of reviews, in this formally define the Characteristic- Review Selection problem. But it can't useful to arbitrary domain.in this they proposed algorithm which provide compact set of reviews but it not consider distribution of positive and negative opinion.

[9]Selecting a comprehensive Set of Reviews, which formulates the review retrieval problem as a maximum analyses problematic and need to select high coverage problems having different view-points and top a maximum number of different features reviewed product(+,-).provide authentic review using TOPQLTY algorithm sorting technique problem is based on limited review set.

[10]Efficient confident search in large review corpora, introduce CREST (Confident Review Search Tool).using this tool e can select a high compact set of review in large corpus

Also. It provides redundancy filtering method. The filtered corpus maintains all the useful information and is considerably smaller, which makes it easier to store and to search. This is user friendly and applicable to large corpus. Problem is this system work on artificial review.

[4]Selecting a diversified set of reviews, this introduce selection product wise means select set of reviews for each product. This process based on different attributes like coverage and opinion diversity.it provide better diversification result especially for selecting smaller sets of review.

[5]Tips, dones and to-dos, Uncovering user profiles in foursquare, in this paper, they analyses how Foursquare users exploit these three features tips, dons and to-dos uncovering

Different behavior profiles. Also provide evidence of spamming, showing the existence of users that post tips whose contents are unrelated to the nature or domain of the venue where the tips were left. Not recover attack actions on Foursquare.

\section{PROPOSED SYSTEM}

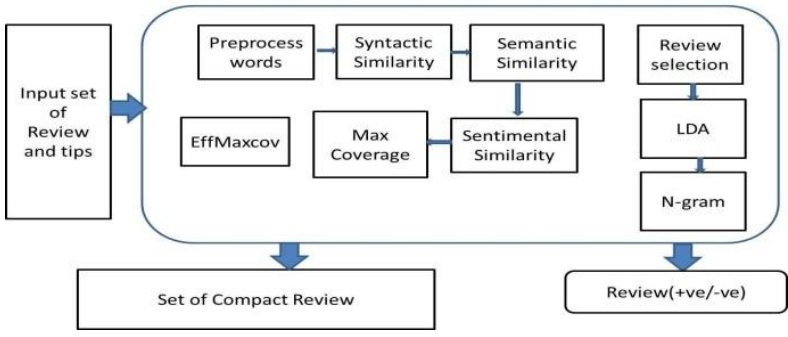

Fig1: Proposed System

\subsection{Syntactic Similarity}

A review sentence and a tip are syntactically similar if they share important keywords or common words. To find Syntactic similarity Proposed system use Cosine Similarity, Extended Jaccard similarity, Euclidean Distance similarity, Dice Similarity measure. After using all this e conclude that cosine similarity is better.

Cosine $=\mathrm{d} 1 * \mathrm{~d} 2 /|\mathrm{d} 1||\mathrm{d} 2|$
Euclidean $=\sqrt{(\mathrm{d} 1-\mathrm{d} 2)+(\mathrm{d} 1-\mathrm{d} 2) \ldots \ldots \ldots}$
Jaccard $=\mathrm{d} 1 * \mathrm{~d} 2 / \mathrm{d} 1 * \mathrm{~d} 1+\mathrm{d} 2 * \mathrm{~d} 2-\mathrm{d} 1 * \mathrm{~d} 2$


Dice $=2 *(\mathrm{~d} 1-\mathrm{d} 2) /(\mathrm{d} 1 * \mathrm{~d} 1)+(\mathrm{d} 2 * \mathrm{~d} 2)$

\subsection{Semantic Similarity}

A sentence and a tip may discuss the same concept (e.g., a menu dish), but use different words (e.g., soup vs. broth). In this case we say that they have high semantic similarity. Latent Dirichlet Allocation: LDA associates each tip $t$ with a probability distribution t over the topics. For each topic as it is learnt from the tips, proposed system can estimate the topic distribution for $\mathrm{T}$ and this topic modeling use for each review sentence $\mathrm{s}$.

\subsection{Sentimental Similarity}

Every opinion having their sentiment which reflects from sentence positive, negative or neutral. Hence, in addition to sharing syntactical similar keywords, semantic similarity of word and concepts, proposed system would also like a matching Review sentence-tip pair to share the same sentiment (positive or negative). Proposed system define the sentiment similarity between a Review sentence $\mathrm{s}$ and a tip $\mathrm{t}$ as the product of their polarities: it approaches 1 when the sentence and the tip polarities are similar; it approaches 1 when their polarities are opposite. It approaches 0 when the tip or the sentence polarity is neutral.

Therefore, proposed system has:

Sentimental Similarity $(s, t)=\operatorname{polarity}(s) *$ polarity $(t)$

And use Stanford unique English dictionary for better s and classification of word and phrases. Sentiments are classify using N-gram analysis learn from English Stanford dictionary.

\subsection{Negative opinion Mining}

In associate with above three modules we also develop module which extract negative opinion reviews and find the reason behind the negative review. For this purpose proposed system first find negative reviews then use LDA to find topics, the find unique words, and form the sentence summary so user can read the cause.

\section{RESULT ANALYSIS}

\subsection{Data set}

In this paper proposed system use dataset for reviews and micro review (tips) in (text) restaurant domain. For this Proposed system select Reviews from yelp.com site and Tips (Micro-reviews) from foursquare.com.Size of review dataset is $211,252 \mathrm{~KB}$ and tips dataset is $1956 \mathrm{~KB}$.Constraint for this is need more reviews than tips for selection purpose. Only those tips are selected hose review are available.

\subsection{Experiments}

\subsubsection{Matching}

For the project Matching between a review sentence and tip is one of the important task or its challenging problem. Our project objective is to select those reviews which are the best match ever for tips. Means we need to achieve quality of coverage. Proposed system use good algorithm which covers reviews which are reflection of tips of the same entity.

In Syntactic Similarity Proposed system use Cosine, Extended Jaccard, Euclidean Distance, Dice Similarity measure and proposed system find that from all these Similarity measure Cosine similarity is best for common word matching. Proposed system use cosine similarity in our project our final result is improved. Table 1 shows the result come for similarity. If proposed system see for no. of reviews selected is 5 result of cosine is precision recall and result of Euclidean is precision recall. But afterword's result of cosine is got improved and provide high recall as compare to other.

Table 1. Result for cosine similarity

\begin{tabular}{|c|c|c|c|}
\hline $\begin{array}{c}\text { Top K } \\
\text { reviews }\end{array}$ & $\begin{array}{c}\text { Cosine } \\
\text { similarity }\end{array}$ & Precision & Recall \\
\hline 3 & 0.33 & 0.78 & .80 \\
\hline 5 & 0.6 & 0.64 & .88 \\
\hline 7 & 0.71 & 0.78 & .86 \\
\hline
\end{tabular}

Table 2: Result for Euclidean distance

\begin{tabular}{|c|c|c|c|}
\hline $\begin{array}{c}\text { Top K } \\
\text { reviews }\end{array}$ & $\begin{array}{c}\text { Euclidean } \\
\text { similarity }\end{array}$ & Precision & Recall \\
\hline 3 & .33 & .75 & .89 \\
\hline 5 & .4 & .80 & .85 \\
\hline 7 & .28 & .82 & .84 \\
\hline
\end{tabular}

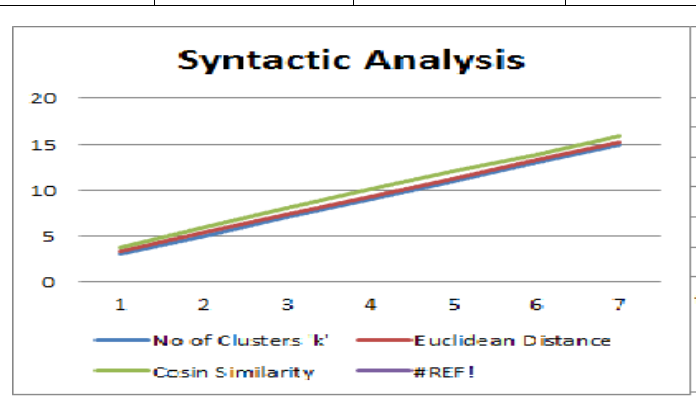

Fig 2: Result of 2 similarity measure

In Semantic Similarity Proposed system use LDA approach which creates different topics using topic modeling for particular tips and these topics are used for match reviews with tips. Proposed system study performance of match is varying if proposed system varies number of topics.

If proposed system compares result of base paper [1] and our project for number of topic 10 then proposed system see in table 3 that our result provide high precision and recall as compare to base paper result. Our system provides more accurate result.

Table 3: Base paper VS Proposed system result for match

\begin{tabular}{|c|c|c|c|c|c|}
\hline \multicolumn{6}{|c|}{ Number Of Topic $=10$} \\
\hline $\begin{array}{l}\text { Precision } \\
\text { (base) }\end{array}$ & $\begin{array}{l}\text { Recall } \\
\text { (base) }\end{array}$ & $\begin{array}{l}\text { Coverable } \\
\text { Tips(base) }\end{array}$ & $\begin{array}{l}\text { Precision } \\
\text { (our) }\end{array}$ & $\begin{array}{l}\text { Recall } \\
\text { (our) }\end{array}$ & $\begin{array}{l}\text { Coverable } \\
\text { Tips(our) }\end{array}$ \\
\hline .80 & .80 & .70 & .100 & .90 & .70 \\
\hline \multicolumn{6}{|c|}{ Base Paper } \\
\hline \multirow{6}{*}{$\begin{array}{r}0.82 \\
0.8 \\
0.78 \\
0.76 \\
0.74 \\
0.72 \\
0.7 \\
0.68 \\
0.66 \\
0.64\end{array}$} & \multicolumn{5}{|c|}{ 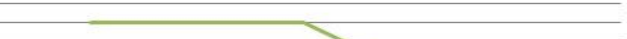 } \\
\hline & \multicolumn{5}{|c|}{ 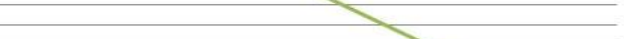 } \\
\hline & \multicolumn{5}{|c|}{ 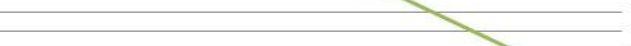 } \\
\hline & \multicolumn{5}{|c|}{ 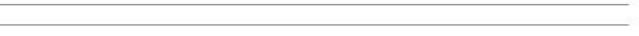 } \\
\hline & \multirow{2}{*}{\multicolumn{2}{|c|}{ Precision (base) }} & Recall(base) & \multirow{2}{*}{\multicolumn{2}{|c|}{ Coverable tips(base) }} \\
\hline & & & Number of Topic $=10$ & & \\
\hline & & & -Series1 & & \\
\hline
\end{tabular}

Fig2:For No.of topic=10 base paper result 


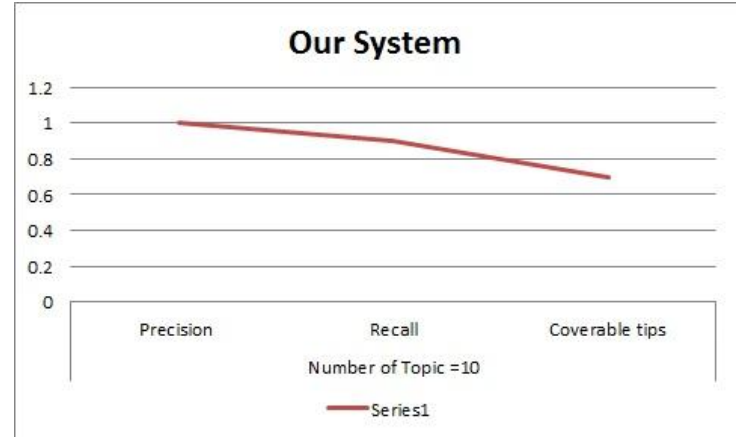

Fig3: For No.of topic=10 Proposed system result

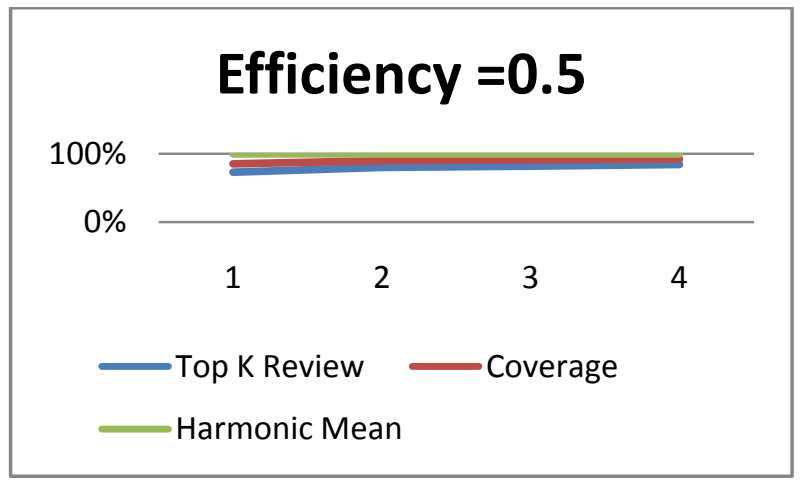

Fig 4:Coverage of top $k$ review

Fig 4 shows that review cover max match tips with consideration of efficiency 0.5

\section{CONCLUSION AND FUTURE SCOPE}

As in this two stage process in fist stage matching process is done and in second process is selecting a compact set of review and provide to user with cause of negative opinion. For this Proposed system performs semantic similarity, sentimental similarity, syntactic similarity, which give high coverage of reviews. Even if high coverage, efficiency is low.so to select high coverage and high efficiency use greedy effmaxcov algorithm. Standford unique English dictionary of negative word is maintain, which provide a way to find negative opinion reasons. Project is developing to improve speed of matching similarity between review and microreview.Provide user a set of compact reviews. In future this system we can develop for short tweet also. And if system is get develop in parallel computing domain then surely it provides high speed computation.

\section{ACKNOWLEDGMENTS}

First and foremost, I would like to thank my guide for her guidance and support. I would also like to thank to my friends for listening my ideas, asking questions and providing feedback and suggestions for improving ideas.

\section{REFERENCES}

[1] Thanh-Son Nguyen, Hady W. Lauw, Member, IEEE, andPanayiotis Tsaparas, Member, IEEEReview Selection UsingMicro-Reviews in IEEE TRANSACTIONS ON KNOWLEDGE AND DATA ENGINEERING, VOL. 27, NO. 4, APRIL 2015.
[2] E. Kouloumpis, T. Wilson, and J. Moore, Twitter sentiment analysis: The good the bad and the omg, in 5th Int. Conf.Weblogs Social Media., 2011, pp. 538541. 1110 .

[3] Q. Yuan, G. Cong, Z. Ma, A. Sun, and N. M Thalmann,Timeaware point-of-interest recommendation, in Proc. $36^{\text {th }}$ Int. ACM SIGIR Conf. Res. Develop. Inf. Retrieval., 2013, pp.363372.

[4] W. Yu, R. Zhang, X. He, and C. Sha, Selecting a diversified set of reviews, in Proc. 15th Asia-Pacific Web Conf, Jun. 2013, pp. 31663173.

[5] M. A. Vasconcelos, S. Ricci, J. Almeida, F. Benevenuto, and V. Almeida, Tips, dones and todos: Uncovering userprofiles in foursquare, in in Proc. 5th ACM Int. Conf. Web Search Data Mining, 2012, pp. 653662.

[6] K. Ganesan, C. Zhai, and E. Viegas, Micropinion generation:An unsupervised approach to generating ultraconcise summaries of opinions, in Proc. 21st Int Conf. World Wide Web., 2012, pp. 869878 SIGCHI Conference on Human Factors in Computing Systems

[7] T. Lapps, M. Cornella, and E. Teri, Selecting a characteristic set of reviews, in Proc. 18th ACM SIGKDD Int. Conf. Know.Disco. Data Mining, 2012, pp. 832840

[8] P. Sinha, S. Mehrotra, and R. Jain, Summarization of personal photologs using multidimensional content and context, in Proc. 1st ACM Int. Conf. Multimedia Retrieval, 2011.

[9] P. Tsiaris, A. Ntoulas, and E. Terzi, Selecting a comprehensiveset of reviews, in Proc. 17th ACM SIGKDD Int. Conf. Knowl. Discov. Data Mining. 2011, pp. 168176

[10] T. Lappas and D. Gunopulos, Efficient confident search in large review corpora, in Proc. Eur. Conf. Mach. Learn. Knowl. Discovery Databases: Part II., 2010, pp. 195210

[11] K. Ganesan, C. Zhai, and J. Han, Opinosis: A graphbasedapproach to abstractive summarization of highly redundant opinions, in Proc. 23rd Int. Conf. Comput. Linguistics. 2010, pp. 340348.

[12] Y. Lu, P. Tsaparas, A. Ntoulas, and L. Polanyi, Exploiting social context for review quality prediction, in Proc. $19^{\text {th }}$ Int. Conf. World Wide Web., Jun. 2009, pp. 15971604.

[13] B. J. Jansen, M. Zhang, K. Sobel, and A. Chowdury, Twitter power: Tweets as electronic word of mouth, in J.Amer. Soc. Inf. Sci. Technol.,, vol. 60, no. 11, pp. 21692188,2009 .

[14] A. Ghose and P. G. Ipeirotis, Designing novel reviewranking systems: Predicting the usefulness and impact of reviews, in Proc. 9th Int. Conf. Electron.Commerce 2007. 